\title{
Distribution Based Representative Sets for Multi-objective Integer Programs
}

\author{
Sami Serkan Özarık ${ }^{\mathrm{a}}$, Banu Lokman ${ }^{\mathrm{b}, \mathrm{c}, *}$, Murat Köksalan ${ }^{\mathrm{c}, \mathrm{d}}$ \\ ${ }^{a}$ Eindhoven University of Technology, Pav. F08, Eindhoven NL 5600 MB, The Netherlands \\ ${ }^{b}$ Portsmouth Business School, Centre of Operations Research and Logistics (CORL), \\ University of Portsmouth, Portsmouth, PO1 3DE, United Kingdom \\ ${ }^{c}$ Department of Industrial Engineering, Middle East Technical University, 06800, Turkey \\ ${ }^{d}$ Ross School of Business, University of Michigan, Ann Arbor, Michigan 48109, USA
}

\begin{abstract}
We study and exploit the characteristics of the nondominated sets of Multiobjective Integer Programs (MOIPs). We introduce a density measure and search for common properties of the distributions of nondominated points for different MOIPs. We design a procedure that categorizes the nondominated set into regions based on the densities of nondominated points. We develop an approach that generates representative sets of nondominated points using the estimated density information in different regions for general MOIPs. Experiments show that our approach is robust across different types of MOIPs.
\end{abstract}

Keywords: nondominated point, representative set, multi-objective integer programs, density-based quality measure.

\section{Introduction}

Multi-objective Integer Programs (MOIPs) are widely used due to their capabilities to represent a variety of practical decision problems. The objectives are typically conflicting in nature. An efficient solution has the property that there exists no feasible solution that improves each objective simultaneously; one needs to sacrifice from some objectives in order to improve others. The

\footnotetext{
* Corresponding author

Email addresses: s.s.ozarik@tue.nl (Sami Serkan Özarı), banu.lokman@port.ac.uk (Banu Lokman), koksalan@metu.edu.tr (Murat Köksalan)
} 
image of the set of efficient solutions in objective function space is known as the set of nondominated points.

As the problem size increases in the MOIPs, typically the nondominated set gets larger. Finding a single nondominated point is computationally demanding for large-sized MOIPs. Many metaheuristic and heuristic approaches have been developed for MOIPs. Ehrgott and Gandibleux (2004) review algorithms developed for Multi-Objective Combinatorial Optimization (MOCO) problems, which are special types of MOIPs.

Several exact algorithms have been developed to find all nondominated points of MOIPs. Sylva and Crema (2004) iteratively generate nondominated points by solving a sequence of progressively more complex single-objective integer problems. Therefore, its computational cost becomes prohibitive for largesized problems. The algorithms developed by Dhaenens et al. (2010) and Przybylski et al. (2010) are based on the two-phase method while Laumanns et al. (2006) and Özlen and Azizoğlu (2009) utilize the epsilon-constraint method. The recent algorithms not only use the properties of the epsilon-constraint method but also systematically decompose the search region to generate new nondominated points at each iteration (see Lokman and Köksalan, 2013, Mavrotas and Florios, 2013, Kirlik and Sayı, 2014, Özlen et al., 2014, Dächert and Klamroth, 2015, Boland et al. 2017, Dächert et al., 2017). Different than these algorithms, Bektaş (2018) defines the search regions using disjunction programming. Although these algorithms could improve the computational time, the main difficulty persists. As Ehrgott and Gandibleux (2000), Ehrgott et al. (2016) and Figueira et al. (2017) discuss in detail, many MOCO problems are hard problems and the number of nondominated points increases with input size. Many of these problems become intractable for even two objectives. Even if all nondominated points can be generated with excessive computational effort, the problem of choosing preferred points still remains. Therefore, generating all nondominated points is neither computationally nor cognitively practical. Instead of generating all nondominated points, finding a subset that represents the entire nondominated set of points well, based on certain quality measures, 
is a practical approach for MOIPs.

Saym (2000) proposes three quality measures to evaluate the representative sets: coverage, uniformity and cardinality. While coverage evaluates how well each nondominated point is represented, uniformity measures how the representative points are distributed over the objective function space. Due to high computational cost of producing each nondominated point, the cardinality measure is important in evaluating the quality of the set. Faulkenberg and Wiecek (2010) review quality measures to assess the discrete representations of the nondominated sets and classify them into three groups as suggested by Sayın (2000).

Sylva and Crema (2007), Masin and Bukchin (2008) and Ceyhan et al. (2019) develop algorithms that generate a representative set for MOIPs. These algorithms allow the decision maker (DM) can stop the algorithm when the desired cardinality or coverage level is achieved. The algorithms developed by Sylva and Crema (2007) and Masin and Bukchin (2008) solve a more-constrained model at each iteration in order to generate a new representative point. Ceyhan et al. (2019) utilize a decomposition and search method in representative generation and provide a substantial improvement over these algorithms. Ceyhan et al. (2019) also develop a holistic approach, Surface projection algorithm (SPA), that is designed to generate a high-quality representative set when the number of representative points is known a priori. Bektaş (2018)'s algorithm also generates a subset of the nondominated set but he does not measure the quality of the representation.

Different regions of the nondominated set are treated in a similar manner in these studies. We have observed on many problems that nondominated points are not uniformly distributed over the nondominated set. Some regions are dense and some are sparse in terms of nondominated points. To the best of our knowledge, the literature in this area has not differentiated between different regions based on the characteristics of the nondominated set. However, the DM could be interested in differential representation of different parts. For example, the DM may want a representation proportional to the nondominated points in 
a region. Alternatively, the DM may want to generate a more than proportionate number of representative points from the sparse regions. Therefore, unlike the existing studies in the literature, we aim to characterize different regions of the nondominated set and use this information to generate a representation of this set. To that end, we define a new performance measure that evaluates the quality of the representative set in conjunction with the distribution of nondominated points in the objective function space. We first introduce a density-based quality measure and analyze typical distributions of nondominated points for different MOIPs. Based on our findings, we develop a solution framework that i) approximates the nondominated set using a surface, ii) categorizes the points into subregions based on their estimated densities, and generates distributionbased representative sets.

In Section 2, we provide background information on MOIPs. In Section 3, we introduce a density measure and analyze the distribution properties of MOIPs. In Section 4, we present an approach that estimates the distribution of the points on the nondominated set and develop an algorithm to generate distributionbased representative sets together with the results of our experiments. We present concluding remarks in Section 5.

\section{Background}

In general, a MOIP can be defined as:

$$
\text { "Max" }{ }_{\mathbf{x} \in \mathbf{X}} f(\mathbf{x})
$$

where $f(\mathbf{x})=\left(z_{1}(\mathbf{x}), z_{2}(\mathbf{x}), \ldots, z_{m}(\mathbf{x})\right)$ is an $m$-dimensional objective function vector, $\mathbf{x}$ is an integer decision vector and $\mathbf{X}$ is the feasible set of decision vectors, $\mathbf{X} \subseteq \mathbb{Z}^{l}$.

Definition 1. A solution $\mathbf{x} \in \mathbf{X}$ is said to be efficient if there does not exist any $\mathbf{x}^{\prime} \in \mathbf{X}$ that satisfies $z_{i}\left(\mathbf{x}^{\prime}\right) \geq z_{i}(\mathbf{x})$ for $i=1, \ldots, m$ and $z_{i}\left(\mathbf{x}^{\prime}\right)>z_{i}(\mathbf{x})$ for at least one $i$.

The set of the efficient solutions is denoted as $\mathbf{X}_{E}$. 
Definition 2. If $\mathbf{x}$ is efficient, $\mathbf{x} \in \mathbf{X}_{E}$, then its image in the objective space, $f(\mathbf{x})$, is said to be a nondominated point.

$\boldsymbol{\Omega}=\left\{\mathbf{z} \mid \mathbf{z}=f(\mathbf{x}), \mathbf{x} \in \mathbf{X}_{E}\right\}$ is the set of all nondominated points. In this study, we assume $\boldsymbol{\Omega}$ is finite and $N=|\boldsymbol{\Omega}|$ represents the cardinality of the nondominated set.

The ideal point, $\mathbf{z}^{I P}=\left(z_{1}^{I P}, z_{2}^{I P}, \ldots, z_{m}^{I P}\right)$, is composed of the best values of each objective, $z_{i}^{I P}=\max _{\mathbf{x} \in \mathbf{X}}\left(z_{i}(\mathbf{x})\right) i=1, \ldots, m$, while the nadir point, $\mathbf{z}^{N P}=$ $\left(z_{1}^{N P}, z_{2}^{N P}, \ldots, z_{m}^{N P}\right)$, comprises the worst objective values in the efficient set, $\mathbf{X}_{E}: z_{i}^{N P}=\min _{\mathbf{x} \in \mathbf{X}_{E}}\left(z_{i}(\mathbf{x})\right) i=1, \ldots, m$.

Since finding all nondominated points is computationally demanding or even prohibitive for large-sized MOIPs, there has been research that aims to find a representative subset of nondominated points. We next define three measures introduced by Saym (2000) to evaluate the quality of a representative set. In our definitions, we use a distance function $d(\cdot, \cdot)$ defined on $\mathbb{R}^{m}$.

Definition 3. Let $\alpha \in \mathbb{R}_{\geq}$and $\mathbf{R} \subseteq \boldsymbol{\Omega}$ be a discrete set. For any $\mathbf{z} \in \boldsymbol{\Omega}$, if there exists $\mathbf{y} \in \mathbf{R}$ satisfying $d(\mathbf{y}, \mathbf{z}) \leq \alpha, \mathbf{R}$ is said to be a $d_{\alpha}$-representation of $\boldsymbol{\Omega}$. Then, the smallest $\alpha$ value,

$$
\alpha_{\mathbf{R}}=\max _{\mathbf{z} \in \mathbf{\Omega}} \min _{\mathbf{y} \in \mathbf{R}} d(\mathbf{y}, \mathbf{z})
$$

is the coverage gap of $\mathbf{R}$.

Here, a representative point, $\mathbf{y} \in \mathbf{R}$, is assigned to each nondominated point, $\mathbf{z} \in \boldsymbol{\Omega}$, based on a distance function. The coverage gap value, $\alpha_{\mathbf{R}}$, corresponds to the distance of the worst represented point to its representative. At the coverage gap calculations, unlike Saym (2000), we use a Tchebycheff-based distance function to quantify the representation error, $\max _{1 \leq i \leq m}\left(z_{i}-y_{i}\right)$ as in Sylva and Crema (2007), Masin and Bukchin (2008) and Ceyhan et al. (2019). That is, the difference between the objective values contributes to the representation error only when the nondominated point is superior to its representative point on that objective, $z_{i}>y_{i}$ for any $i=1, \ldots, m$. Thus, we calculate the coverage 
gap of a representative set $\mathbf{R} \subseteq \boldsymbol{\Omega}$ for a maximization-type MOIP as follows:

$$
\alpha_{\mathbf{R}}=\max _{\mathbf{z} \in \boldsymbol{\Omega}}\left(\min _{\mathbf{y} \in \mathbf{R}}\left(\max _{1 \leq i \leq m}\left(z_{i}-y_{i}\right)\right)\right) .
$$

In addition to coverage, Saym (2000) defines the uniformity and cardinality of the representative set as quality measures to evaluate a discrete representation of the nondominated set. The uniformity measures the minimum distance between representative points and the idea is to have roughly equal spread among them. While coverage gap measures how well each nondominated point is represented, uniformity helps to generate equally-spaced representative points regardless of the actual spacing between the nondominated points. None of these existing measures considers the distribution of the nondominated points over the objective function space. We may be interested in producing representative sets that also reflect the distribution characteristics of the nondominated set. To achieve this, we capture the densities of nondominated points in different regions. We utilize the Parzen Windows Approach (PWA) of Parzen (1962) for this purpose.

\section{Density Estimation with Parzen Windows Approach}

In order to capture the distribution characteristics of the nondominated set, we employ the Parzen Windows Approach (PWA) which is a non-parametric classification method suitable for our purposes. It has been used in pattern recognition, classification, and identification applications (see Duda et al., 1973. pp. 164-177). With the rapid increase in computational power, non-parametric models such Parzen windows estimator have become more manageable to implement. Such methods facilitate to measure the local structure of the data (Vincent and Bengio, 2003). The algorithm developed by Costa and Minisci (2003) also utilizes PWA to approximate the probability density of nondominated points for multi-objective continuous problems and their experiments show that it works well.

PWA measures the density of the nondominated points around any given point $\mathbf{z}$ in the objective function space using a kernel function. The kernel 
function is used to interpolate the contribution of each nondominated point to the density around $\mathbf{z}$. The PWA density value around $\mathbf{z}$ is found using:

$$
P(\mathbf{z})=\frac{1}{h N} \sum_{n=1}^{N} K\left(\frac{d\left(\mathbf{z}^{n}, \mathbf{z}\right)}{h}\right),
$$

where $h$ is the window size (the window width or bandwidth parameter that corresponds to the width of the kernel) and $K($.$) is a kernel function defined on$ $\mathbb{R}^{m}$.

Definition 4. A Kernel is a non-negative real-valued integrable function, K, that satisfies the following conditions:

$$
\begin{gathered}
\int_{-\infty}^{+\infty} K(u) d u=1 \\
K(u)=K(-u) \quad \forall u
\end{gathered}
$$

In our context, kernels define the range and contributions of points around a specific point to the density measure around that point.

\subsection{Distribution Properties of Nondominated Points in Multi-Objective Integer Programs}

In this section, we employ PWA to analyze the distribution properties of MOIPs. We work on MOIPs for each of which the nondominated set is known a priori (see Lokman and Köksalan, 2013, Köksalan and Lokman, 2015). In order to perform density analysis, we first scale the objective values so that they take values between 0 and 1 . Then, we generate equally-spaced hypothetical reference points over the objective function space and measure the density around each such point.

We scale the objective values as in Köksalan and Lokman (2009), $s_{i}=$ $\frac{z_{i}-z_{i}^{I P}}{z_{i}^{N P}-z_{i}^{I P}} i=1, \ldots, m$. Then, we divide the objective function space into hypercubes with equal edge length, $0<e \leq 1$, and the center points of these hypercubes are chosen as reference points, $\mathbf{r}^{j} j=1, \ldots, J$, around which the

density is to be measured (i.e. $J=\frac{1}{e^{m}}$ where $\frac{1}{e}$ is assumed to be a positive 


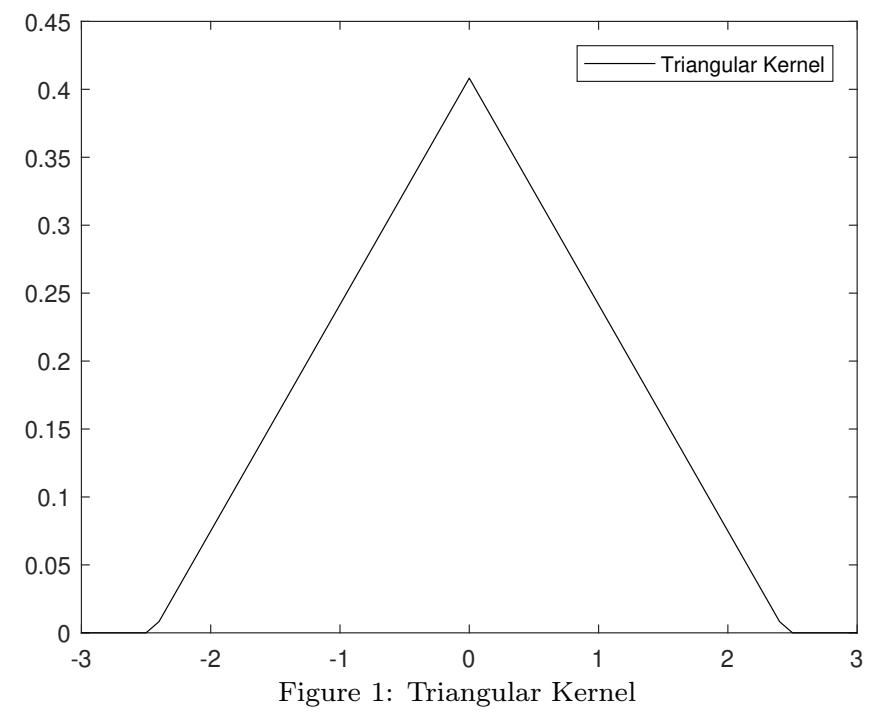

integer). We use the Triangular Kernel Function given in Equation 7 in our density calculations so that the contribution of each nondominated point on the density measure linearly decreases with its distance from the reference point. That is, for each reference point, the closer the nondominated point is, the more it contributes to the density as shown in Figure 1.

$$
K\left(\frac{d\left(\mathbf{s}, \mathbf{r}^{j}\right)}{h}\right)=\left\{\begin{array}{cc}
1-\frac{d\left(\mathbf{s}, \mathbf{r}^{j}\right)}{h / 2}, & \text { if } \frac{d\left(\mathbf{s}, \mathbf{r}^{j}\right)}{h} \leq 1 / 2 \\
0, & \text { otherwise }
\end{array}\right.
$$

After the contribution of each nondominated point is calculated, the next step is to find the total contribution using Equation 8 and measure the density for each reference point using Equation 9 .

$$
\begin{gathered}
P\left(\mathbf{r}^{j}\right)=\sum_{n=1}^{N} K\left(\frac{d\left(\mathbf{s}^{n}, \mathbf{r}^{j}\right)}{h}\right) \\
\bar{P}\left(\mathbf{r}^{j}\right)=\frac{P\left(\mathbf{r}^{j}\right)}{\sum_{j=1}^{J} P\left(\mathbf{r}^{j}\right)}
\end{gathered}
$$

Note that, in contrast with Equation 4, we do not divide total contribution by the number of nondominated points $N$ and the window size $h$ since we fix the values of $h$ and $N$ in our density analysis and normalize the density values 


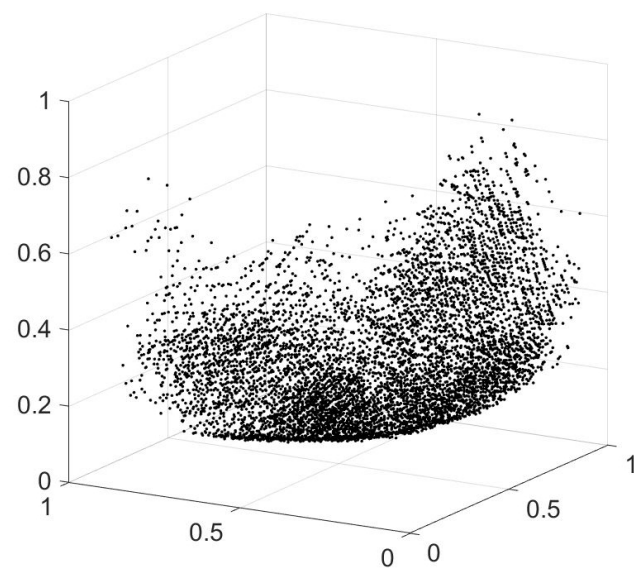

a. The Nondominated Points

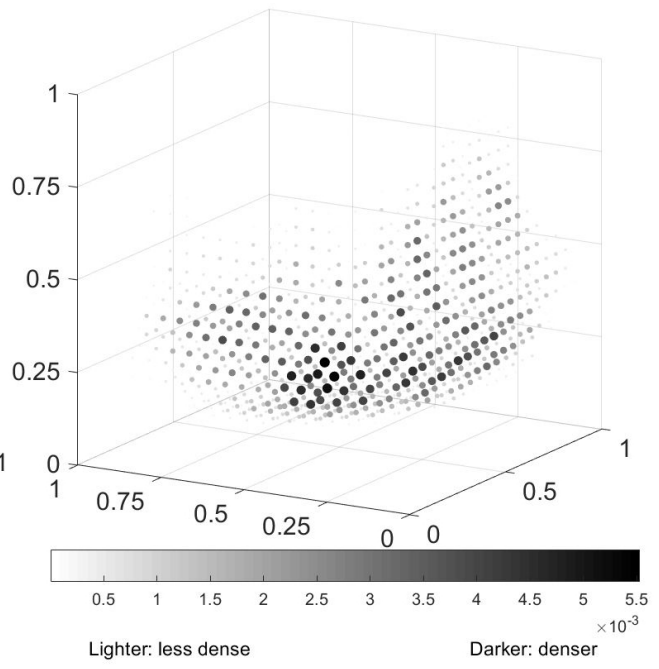

b. PWA Density Values

Figure 2: The nondominated set and PWA density values for a MOAP $(N=6573, h=0.2$,

$$
e=0.05)
$$

in Equation 9 to make them comparable.

To demonstrate, we present the nondominated set and the PWA density values around reference points for a multi-objective assignment problem (MOAP) in Figure 2, In order to generate these reference points, the objective function space is divided into $J=8000$ hypercubes with an edge length of $e=0.05$ units. We specify the window size as $h=0.2$ and use Euclidean distance function in density calculations. In Figure 2, the density values are symbolized with the sizes and colors of the spheres. As the size of each sphere grows and the color changes from gray to black, density increases.

We conduct PWA density analysis on different MOIPs in order to investigate common distribution properties. Figure 3 demonstrates how the density values change over the objective function space for different-sized multi-objective knapsack problems (MOKPs) while Figure 4 compares PWA density values of different types of problems with similar number of nondominated points. In all different settings, the results indicate that the density increases towards the center and the density values approach zero in the vicinity of the points having 


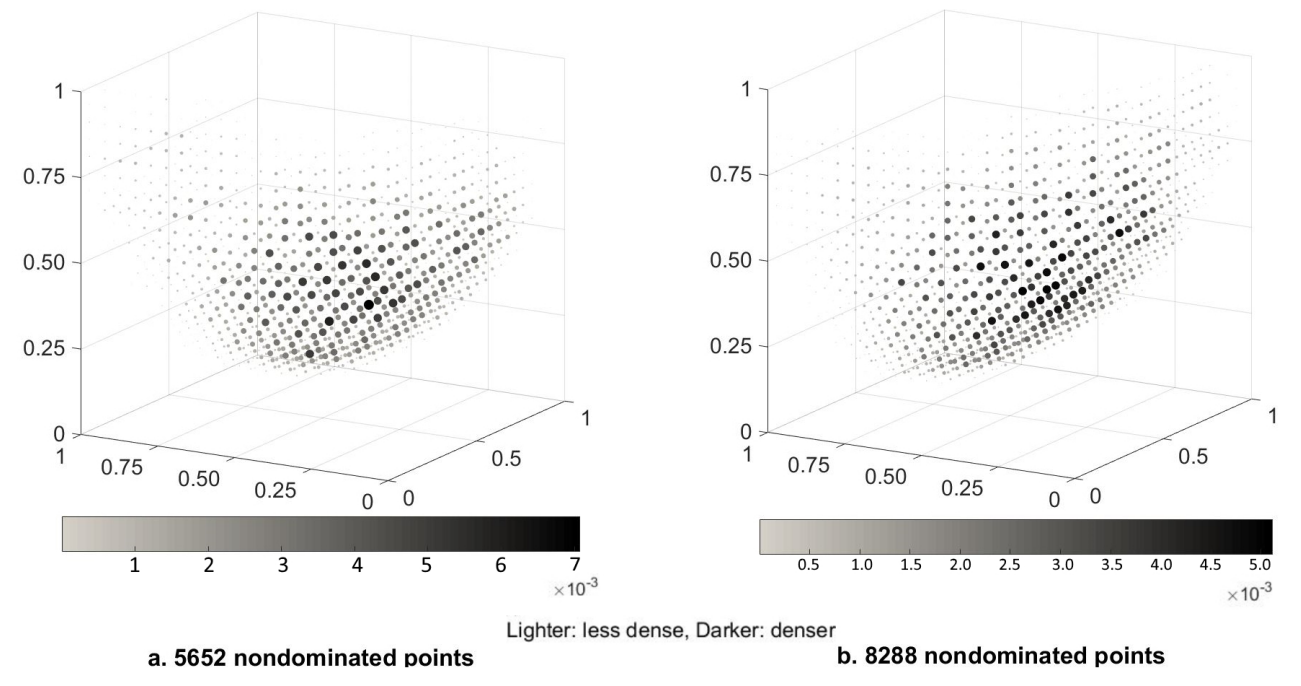

Figure 3: PWA density values for 3-objective 100-item MOKPs with 5652 and 8288 nondominated points, respectively $(h=0.2, e=0.05)$

ideal or nadir point values in at least one of the objectives. For the sake of brevity, we will refer to such points as "extreme" points throughout the paper.

Although PWA chooses equidistant reference points in the objective function space to calculate the density values, we observe in Figures 3 and 4 that nondominated points form a frontier and the rest of the objective function space does not contain nondominated points. Thus, density values at many reference points are zero. If one can estimate the possible locations of the nondominated points, the computational effort can be reduced by eliminating unnecessary density calculations. We next present a procedure to approximate the nondominated set and then choose reference points on the approximated set in the PWA Density Analysis.

\subsection{PWA Density Analysis on the $L_{p}$ surfaces}

Köksalan (1999) estimates the nondominated frontier by fitting an $L_{p}$ curve to bicriteria scheduling problems. Later, Köksalan and Lokman (2009) generalize this approach to approximate the nondominated frontiers of MOCO problems with any number of criteria. Lokman and Köksalan (2014) employ this 

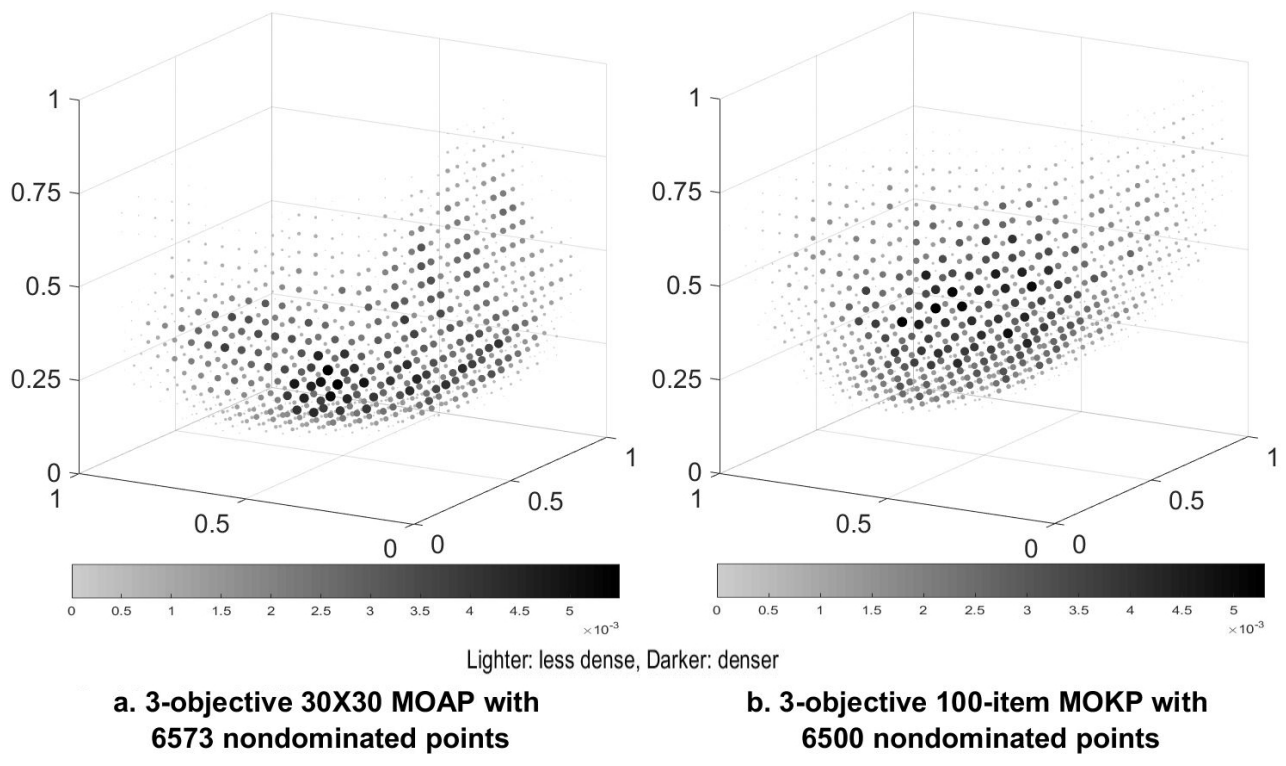

3-objective 30X30 MOAP with

b. 3-objective 100-item MOKP with 6500 nondominated points

Figure 4: PWA density values for MOAP and MOKP with similar number of nondominated

$$
\text { points }(h=0.2, e=0.05)
$$

approximation procedure to define the regions preferred by the DM. Karasakal and Köksalan (2009) generate a discrete representation of the nondominated frontier in multi-objective linear programs using an $L_{p}$ surface. Computational experiments in these studies show that using $L_{p}$ surfaces to approximate the nondominated frontiers performs well. Thus, in this study, we approximate the nondominated frontiers of MOIPs fitting a $L_{p}$ surface:

$$
L_{p}=\left\{\mathbf{s} \in \mathbb{R}_{\geq}^{m}:,\left[\sum_{i=1}^{m}\left(1-s_{i}\right)^{p}\right]^{1 / p}=1 \quad p>0\right\}
$$

Similar to the methodology of Köksalan and Lokman (2009), we fit a $L_{p}$ surface that passes through a "central" point that is at the minimum Tchebycheff distance to the ideal point.

Once the $L_{p}$ surface is obtained, we restrict our density analysis to this surface assuming that it approximates the nondominated frontier well. More specifically, we divide the objective function space into hypercubes with an edge length of $e$ and choose the center of hypercubes as our reference points but 
limit our analysis to the reference points that are close to the $L_{p}$ surface. To do this, for each $\mathbf{r}^{j}$, we find a point, $\mathbf{q}^{j}$, on the $L_{p}$ surface that is at minimum Euclidean distance by solving $\boldsymbol{P}_{\mathbf{1}}(\boldsymbol{j})$.

$\left(P_{1}(j)\right)$

$$
\begin{array}{ll}
\min & d\left(\mathbf{q}^{j}, \mathbf{r}^{j}\right)=\sqrt{\sum_{i=1}^{m}\left(r_{i}^{j}-q_{i}^{j}\right)^{2}} \\
\text { s.t. } & \sum_{i=1}^{m}\left(1-q_{i}^{j}\right)^{p}=1 \\
& 0 \leq q_{i}^{j} \leq 1 \quad i=1, \ldots, m
\end{array}
$$

If $d\left(\mathbf{q}^{j}, \mathbf{r}^{j}\right)>\frac{e}{2}$, the reference point, $\mathbf{r}^{j}$, is excluded from our density analysis and we estimate the density around $\mathbf{r}^{j}$ as zero. Otherwise, we include the corresponding point, $\mathbf{q}^{j}$, on the $L_{p}$ surface to our hypothetical reference point set, $\mathbf{Q}=\left\{\mathbf{q}^{j}: d\left(\mathbf{q}^{j}, \mathbf{r}^{j}\right) \leq \frac{e}{2} \quad j=1, \ldots, J\right\}$. After we obtain the hypothetical reference point set, we conduct a density analysis at these points using PWA.

Figure 5 presents the nondominated set and PWA density values at hypothetical reference points for a three-objective knapsack problem. Working with an $L_{p}$ surface eliminates the unnecessary computational efforts in the regions that do not contain nondominated points. As shown by Köksalan and Lokman (2009), $L_{p}$ surface approximates the nondominated set well in dense regions while the quality of the approximation somewhat deteriorates towards the hypothetical points for which $s_{i}=0$ for some $1 \leq i \leq m$ and $s_{l}=1$ for all $l \neq i$. We refer to these points as "extreme hypothetical points". Since the density also decreases substantially at these extreme points, $L_{p}$ surface provides a good estimate for MOIPs' density calculations.

We perform density analysis on $L_{p}$ surfaces of 100 different MOIPs. The problems included in the analysis are MOAP, MOKP, multi-objective spanning tree problem (MOSTP), and multi-objective shortest path problem (MOSPP). The number of nondominated points in these problems varies between 101 and 


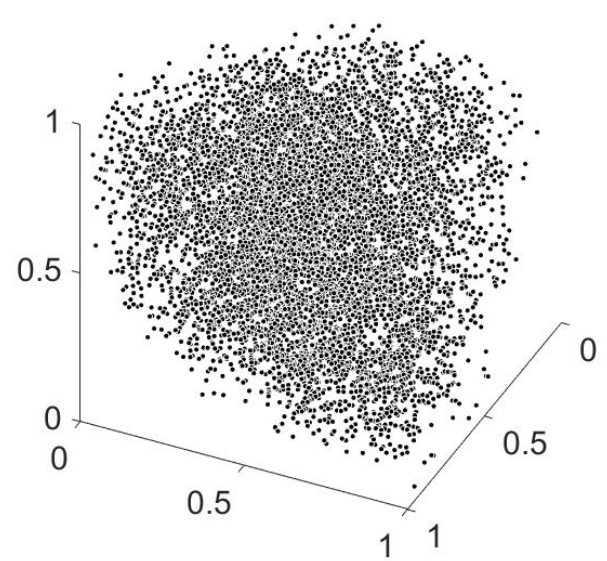

a. The nondominated set
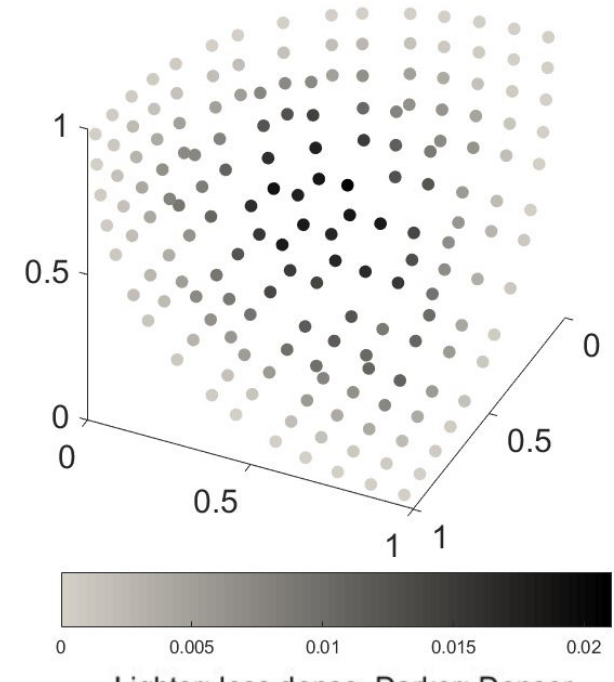

Lighter: less dense, Darker: Denser

\section{b. PWA density values at hypothetical reference point set}

Figure 5: The results for a 3-objective 100-item MOKP $(N=6500, h=0.2, e=0.1)$

10,701 . We observe that there is more variation and a general pattern is less visible when the number of nondominated points is small as shown in Figure 6 . When the number of nondominated points is sufficiently large (more than about $1,000)$, density distributions of different MOIPs tend to have common properties. Figure 7 compares how the density changes over the objective function space for MOAPs with 103 nondominated points and a MOKP having 6500 nondominated points where density increases as the color changes from gray to black. The results show that the most dense point is located around the "center" of the surface and as one moves on the $L_{p}$ surface from the "central point" towards the peripheries, density values of hypothetical points decrease. These features are common not only for different-sized problems but also for different types of MOIPs as demonstrated in Figure 8 . 


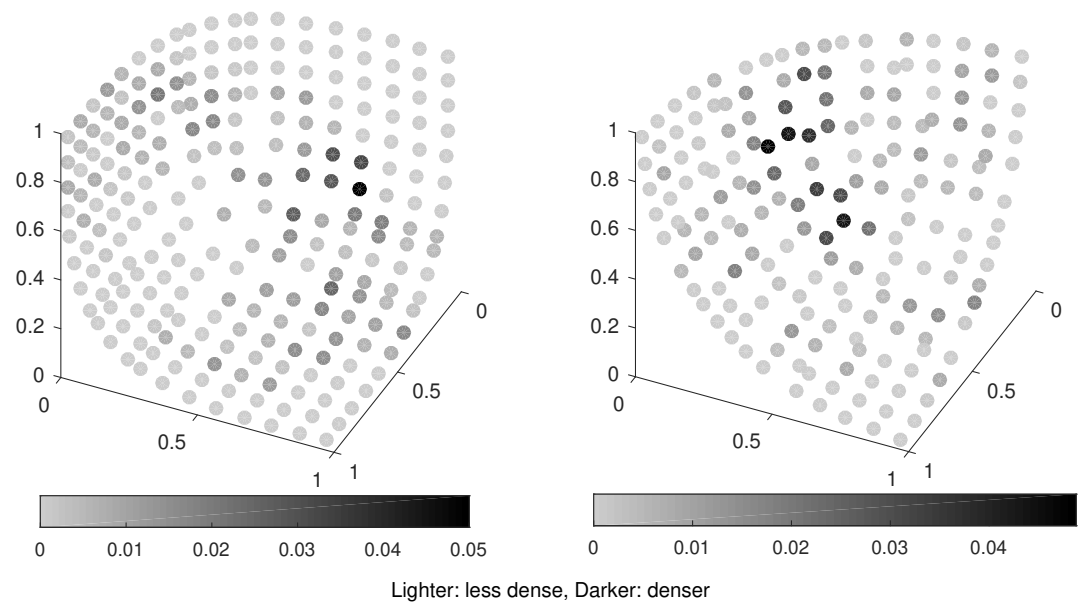

a. 103 nondominated points

b. 107 nondominated points

Figure 6: PWA density values at hypothetical reference point set $(h=0.2, e=0.1)$
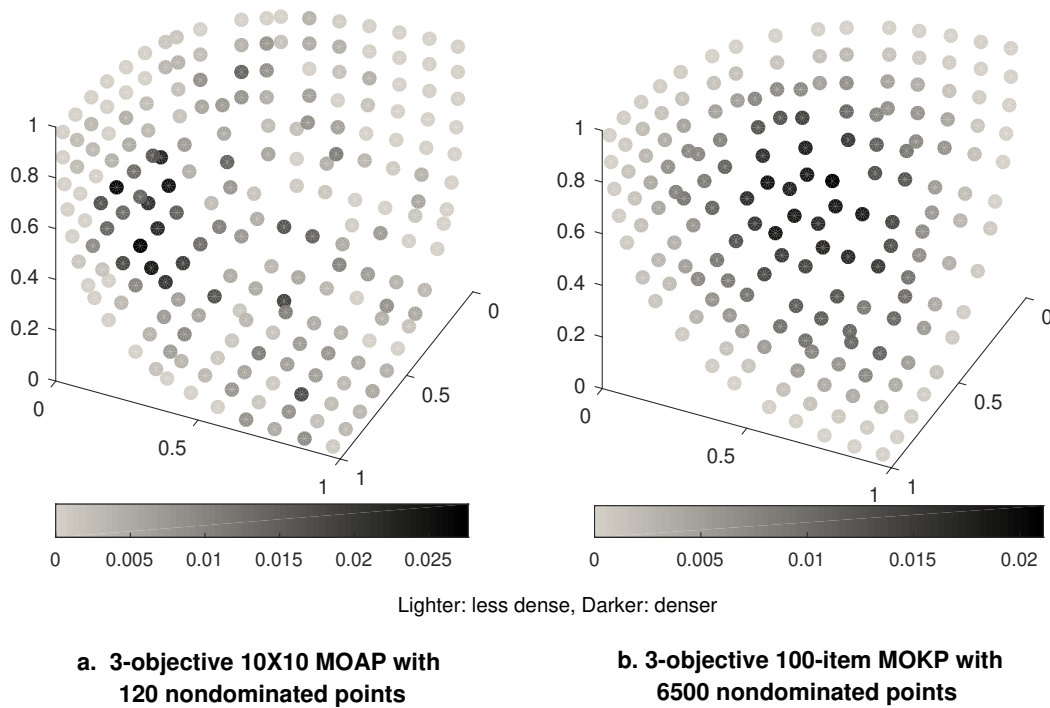
120 nondominated points

b. 3-objective 100-item MOKP with 6500 nondominated points

Figure 7: PWA density estimates at hypothetical reference point sets for different-sized problems $(h=0.2, e=0.1)$ 


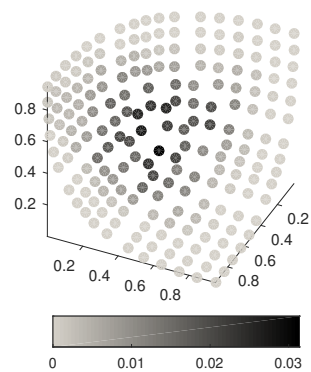

a. 100-item MOKP with 3084 nondominated points
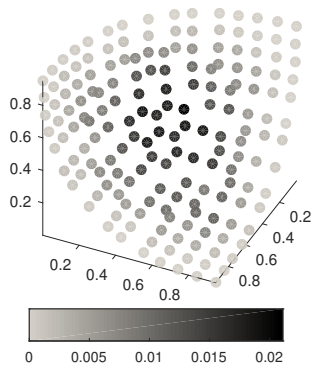

Lighter: less dense, Darker: denser

b. 100 -item MOKP with 6500 nondominated points

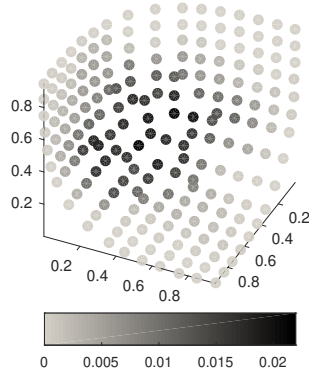

c. $30 \times 30$ MOAP with 6178 nondominated points

Figure 8: PWA density values at a hypothetical reference point sets for 3-objective problems

$$
(h=0.2, e=0.1)
$$

\subsection{Density Based Categorization of Hypothetical Points}

Although there are a number of algorithms in the literature that are designed to find or approximate the nondominated sets, the distribution properties of the nondominated sets have not been studied. Therefore, we utilize our findings in Section 3.2 and develop an approach to categorize hypothetical points according to their estimated density values. In order to capture the distribution properties and generalize the results for MOIPs, we exclude problems having fewer than 1000 nondominated points based on our observation that variations from common properties are high in these problems. However, this is not a drawback because finding representative nondominated points and estimating the densities of such points are important for large-size problems that have many nondominated points.

To classify nondominated points into $K$ categories $C_{1}, \ldots, C_{K}$, we use hypothetical points as our reference points and analyze the densities around them. We observe that there is a large proportion of nondominated points close to a small proportion of hypothetical points and there are a few nondominated points in the neighborhood of a large proportion of hypothetical points, reminiscent of the Pareto rule. Based on this observation, we devise a Pareto rule-like categorization to classify the hypothetical points. After we calculate the scaled 
densities around well-distributed hypothetical points on the fitted $L_{p}$ surface, we sort these hypothetical points in a descending order of their densities. For two categories, $K=2$, while $25 \%$ of the densest hypothetical points account for $70 \%$ of total density, the remaining $75 \%$ of the hypothetical points capture only $30 \%$ of total density as shown in Table 1 .

Table 1: Average percentage of points in each category, $K=2$

\begin{tabular}{ccc}
\hline $\begin{array}{c}\text { Problem } \\
\text { Type }\end{array}$ & $\begin{array}{c}\text { Category 1 } \\
\mathbf{( 7 0} \boldsymbol{\%} \text { of total density })\end{array}$ & $\begin{array}{c}\text { Category 2 } \\
\mathbf{( 3 0} \boldsymbol{\%} \text { of total density })\end{array}$ \\
\hline MOAP & $26 \%$ & $74 \%$ \\
MOSPP & $25 \%$ & $75 \%$ \\
MOKP & $24 \%$ & $76 \%$ \\
\hline Overall & $25 \%$ & $75 \%$ \\
Average & &
\end{tabular}

Similarly, for $K=3$, Table 2 shows that while only $11 \%$ of the densest hypothetical points account for $40 \%$ of total density, $14 \%$ of the next dense hypothetical points contribute $30 \%$, and remaining set contributes $30 \%$ to total density. We classify Category 1 as the high-density region, Categories 2 and 3 as the medium- and low-density regions, respectively. Figure 9 demonstrates the categorization of a MOKP.

Although we use specific percentages based on our observations for the underlying problems, it is possible to use other percentages as desired. It seems from our experiences, however, that a Pareto-like structure is present in the nondominated sets of MOIPs. This structure can be utilized in differentiating between different regions when deciding on how to distribute the representative points.

\subsection{Density-based approximation algorithm (DAA)}

In line with these findings, we design a density-based approximation algorithm (DAA) for MOIPs for which the nondominated sets are assumed to be unknown. The aim of the algorithm is to generate a set of points that approxi- 
Table 2: Average percentage of points in each category, $K=3$

\begin{tabular}{cccc}
\hline $\begin{array}{c}\text { Problem } \\
\text { Type }\end{array}$ & $\begin{array}{c}\text { Category } \mathbf{1} \\
\mathbf{( 4 0} \boldsymbol{\%} \text { of total density })\end{array}$ & $\begin{array}{c}\text { Category } \mathbf{2} \\
\mathbf{( 3 0} \boldsymbol{\%} \text { of total density) }\end{array}$ & $\begin{array}{c}\text { Category } \mathbf{3} \\
\mathbf{( 3 0} \mathbf{\%} \text { of total density })\end{array}$ \\
\hline MOAP & $11 \%$ & $15 \%$ & $74 \%$ \\
MOSPP & $11 \%$ & $14 \%$ & $75 \%$ \\
MOKP & $10 \%$ & $14 \%$ & $76 \%$ \\
\hline Overall & $11 \%$ & $14 \%$ & $75 \%$ \\
Average & & &
\end{tabular}

mates the nondominated set and to estimate their categories in relation to the densities of nondominated points around them. The algorithm first fits an $L_{p}$ surface using a central point as in Köksalan and Lokman (2009) and generates hypothetical reference points on this surface. Since the nondominated set is unknown to the algorithm, the actual densities of the hypothetical points are not known. Assuming that the densest point is the "central" point and the density decreases as the distance from the central point increases, we categorize hypothetical points as follows:

- $11 \%$ of the hypothetical points that are in the densest region (close to the central point) are in Category 1 covering an estimated $40 \%$ of total density.

- Next densest $14 \%$ of the hypothetical points are in Category 2 covering an estimated $30 \%$ of total density.

- Remaining $75 \%$ of the hypothetical points are in Category 3 covering an estimated $30 \%$ of total density.

In order to evaluate the performance of DAA, we compare the true density categories of each hypothetical point with those estimated by DAA. Figure 8 shows the comparison of true and estimated categories of hypothetical points for a MOKP. The results for 51 different MOIPs show that our estimation method assigns $87 \%$ of the hypothetical points to the their correct categories on average. For the remaining hypothetical points, the deviation of the estimated category 

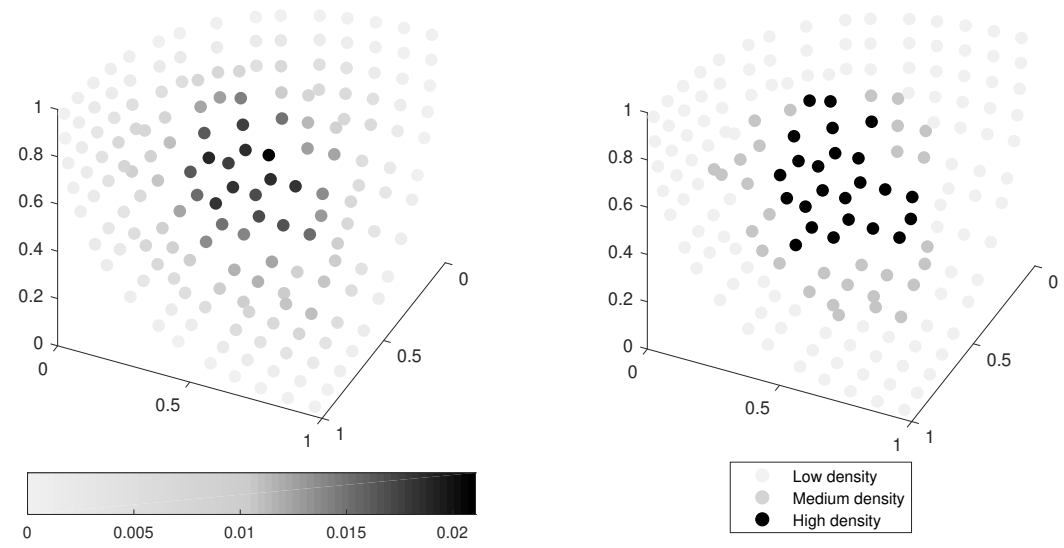

Lighter: less dense, Darker: denser

a. PWA density values at hypothetical reference point set

b. Categorization based on PWA density values

Figure 9: PWA density values and categorization for a 3-objective 100-item MOKP

$$
(N=6500, K=3, h=0.2, e=0.1)
$$

from the true category is not more than one level. $6 \%$ are assigned to a less dense category and $7 \%$ to a denser category than they actually belong to. None of the points in the densest category are assigned to the least dense category and vice verse. After generating the nondominated points at the extreme points and a central nondominated point, DAA successfully estimates the densities of nondominated points in different regions.

\section{Distribution-Based Representation For Multi-objective Integer Pro- grams}

Although DAA is capable of approximating the nondominated set of MOIPs with a set of hypothetical points with density information, there is no guarantee that these points would be nondominated or feasible. Finding true nondominated points as representative points would be more desirable for DMs. We can use the results of DAA and search for true nondominated representative 


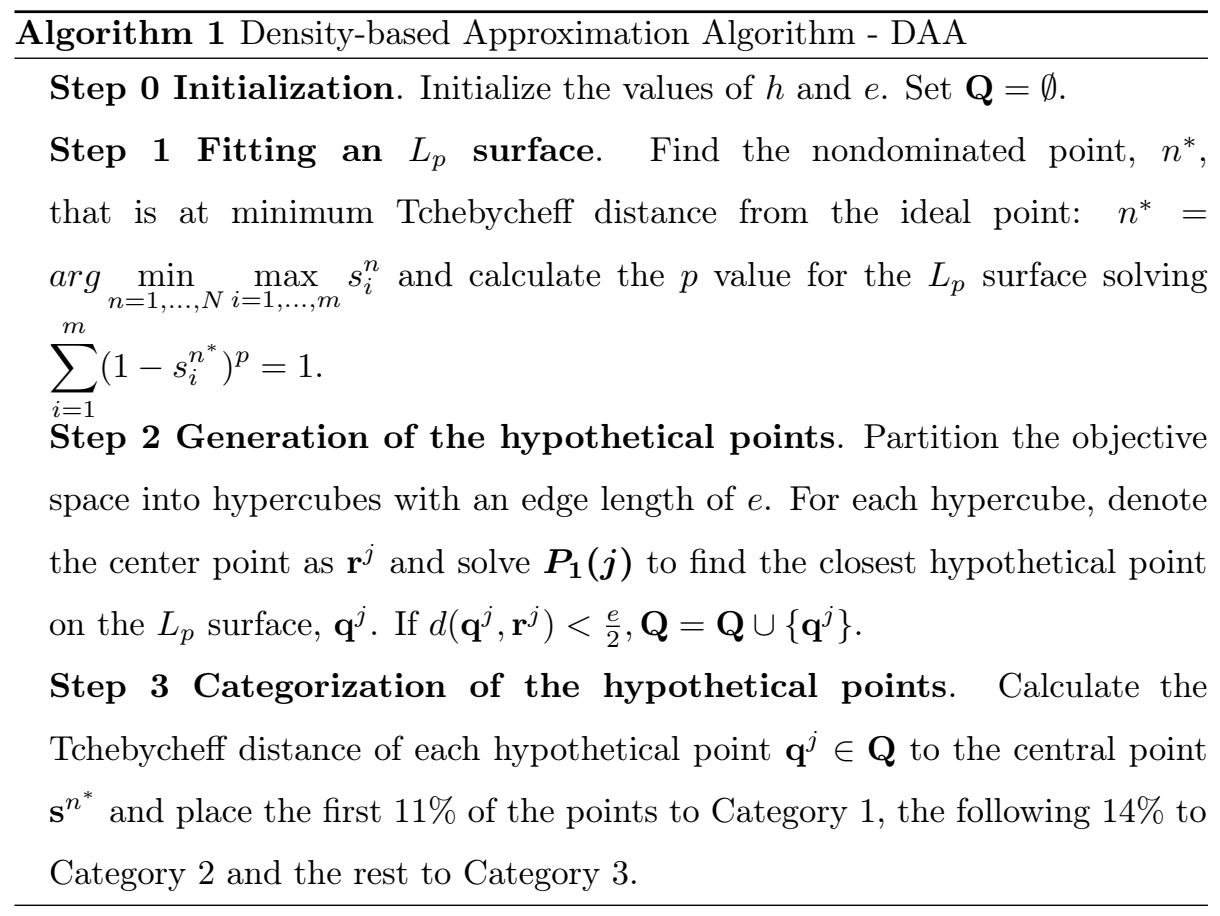

points that satisfy desirable properties based on the application of interest. For example, we may wish to generate relatively more representative points from the denser regions or conversely we may wish to positively discriminate the less dense regions depending on the specific application of interest. In order to take the distribution of nondominated points into consideration, we define a new quality measure, which we call weighted coverage gap. We utilize the coverage gap measure used by Masin and Bukchin (2008), and introduce weights to allow for differentiation between regions that have different properties (densities in our case).

Definition 5. Let $\alpha(\boldsymbol{\lambda}) \in \mathbb{R}_{\geq}$and $\mathbf{R} \subseteq \boldsymbol{\Omega}$ be a discrete set that represents $\boldsymbol{\Omega}$. Let $0 \leq \boldsymbol{\lambda}(\mathbf{z}) \leq 1$ be the weight value associated with the region where point $\mathbf{z}$ lies. If there exists $\mathbf{y} \in \mathbf{R}$ satisfying $\boldsymbol{\lambda}(\mathbf{z}) d(\mathbf{y}, \mathbf{z}) \leq \alpha(\boldsymbol{\lambda})$ for any $\mathbf{z} \in \boldsymbol{\Omega}, \mathbf{R}$ is said to be $d_{\alpha(\boldsymbol{\lambda})}$-representation of $\boldsymbol{\Omega}$. Then, the smallest $\alpha(\boldsymbol{\lambda})$ value,

$$
\alpha_{\mathbf{R}}(\boldsymbol{\lambda})=\max _{\mathbf{z} \in \mathbf{\Omega}} \min _{\mathbf{y} \in \mathbf{R}} \boldsymbol{\lambda}(\mathbf{z}) d(\mathbf{y}, \mathbf{z}),
$$




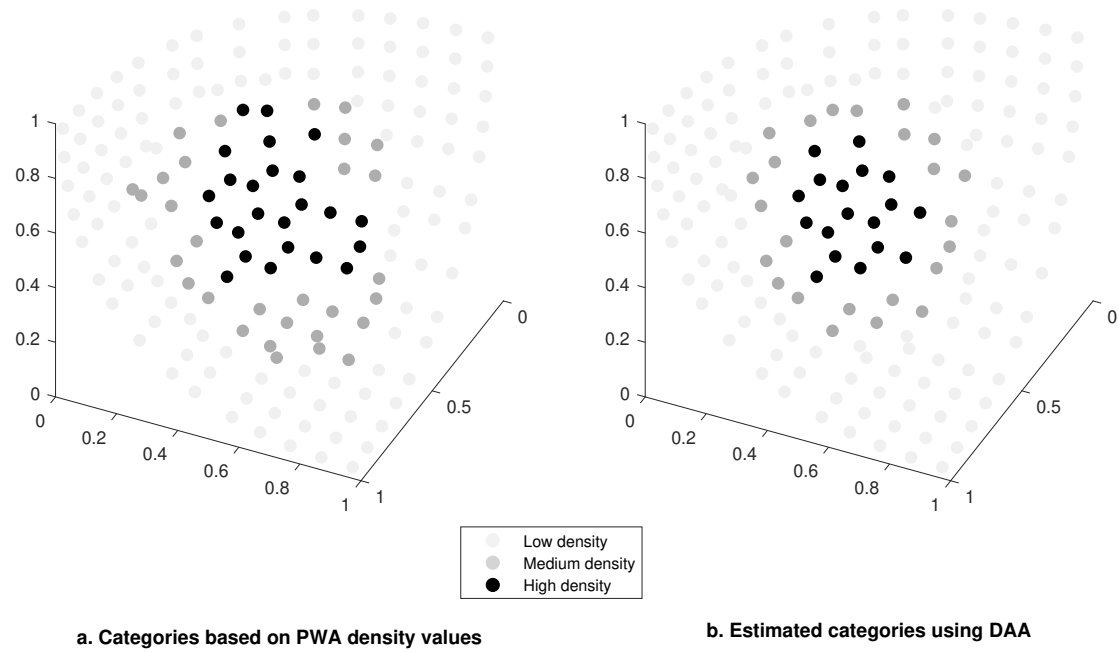

Figure 10: Categorization of the hypothetical points for a 3-objective 100-item MOKP

$$
(N=6500, K=3, h=0.2, e=0.1)
$$

is the weighted coverage gap of set $\mathbf{R}$.

As in the calculations for the coverage gap value, a representative point $\mathbf{y} \in \mathbf{R}$ is assigned to each nondominated point $\mathbf{z} \in \boldsymbol{\Omega}$ but $\alpha(\boldsymbol{\lambda})$ corresponds to the maximum weighted distance between the nondominated points and their representatives. Therefore, the quality of the coverage of each nondominated point $\mathbf{z} \in \boldsymbol{\Omega}$ depends on the selection of $\boldsymbol{\lambda}(\mathbf{z})$.

We scale the objective function values and transform the feasible set $\Omega$ in the objective function space to $\boldsymbol{\Omega}^{\prime}$. We use the Tchebycheff-based distance function:

$$
\alpha_{\mathbf{R}}(\boldsymbol{\lambda})=\max _{\mathbf{s} \in \boldsymbol{\Omega}^{\prime}}\left(\min _{\mathbf{y} \in \mathbf{R}}\left(\max _{1 \leq q \leq m}\left(\boldsymbol{\lambda}(\mathbf{s})\left(s_{i}-y_{i}\right)\right)\right)\right)
$$

We associate the weight values with the categories $C_{1}, \ldots, C_{K}$ and hence 
define $\boldsymbol{\lambda}(\mathbf{s})$ based on the density level around $\mathbf{s}$ :

$$
\boldsymbol{\lambda}(\mathbf{s})=\left\{\begin{array}{ccc}
\lambda_{1} & \text { if } & \mathbf{s} \in C_{1} \\
\lambda_{2} & \text { if } & \mathbf{s} \in C_{2} \\
\ldots & & \\
\lambda_{K} & \text { if } & \mathbf{s} \in C_{K}
\end{array}\right.
$$

The values of $\boldsymbol{\lambda}_{1}, \ldots, \boldsymbol{\lambda}_{K}$ can be set to represent different concerns toward the properties of categories. For instance, we may improve the representation quality in dense regions by assigning higher weight values to the points in Category 1 . We may assign a weight value of zero to low-density regions if we only wish to represent regions with higher density. It is possible to take into account different concerns by assigning different weights to different regions.

\subsection{A Distribution-based Representation Algorithm - DBA}

In order to generate a set of true nondominated points to meet a desired weighted coverage gap level for a prespecified weight vector, we develop a distribution-based representation algorithm (DBA). In DBA, we first apply DAA to find a hypothethical point set, $\mathbf{Q}$, that approximates the nondominated frontier and estimate their density levels. We next solve $\boldsymbol{P}_{\mathbf{2}}$ that finds a minimum cardinality $d_{\alpha(\lambda)}$-representation of $\mathbf{Q}$.

$\left(P_{2}\right)$ :

$$
\min \sum_{j=1}^{|\mathbf{Q}|} v_{j}
$$

$$
\begin{array}{rlrl}
\text { s.t. } \quad\left(q_{i}^{t}-q_{i}^{j}\right) \boldsymbol{\lambda}_{i} u_{t j} & \leq \alpha(\boldsymbol{\lambda}) & \forall i, j, t \\
\sum_{t=1}^{|\mathbf{Q}|} u_{t j} & \leq|\mathbf{Q}| v_{j} & \forall j \\
\sum_{j=1}^{|\mathbf{Q}|} u_{t j} & =1 & \forall t \\
v_{j}, u_{t j} & \in\{0,1\} \quad i=1, \ldots, m & j, t=1, \ldots,|\mathbf{Q}|
\end{array}
$$



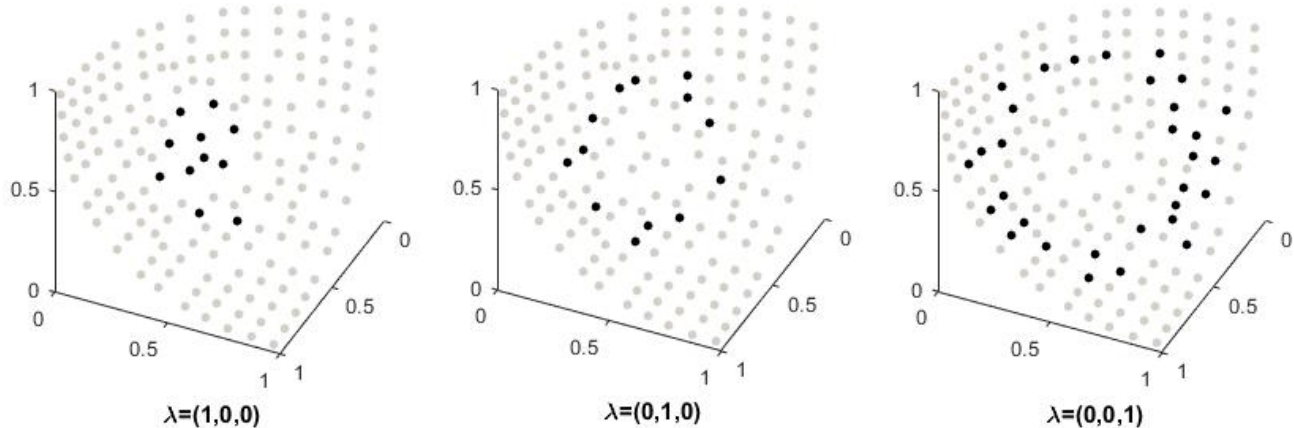

- Hypothetical Points

Representative Hypothetical Points

Figure 11: Representative point selection among the hypothetical points for a 3-objective 100-item MOKP with 3198 nondominated points

In $\left(\boldsymbol{P}_{\mathbf{2}}\right)$, the binary decision variable $v_{j}$ takes a value of 1 if the hypothetical point $\mathbf{q}^{j}$ is selected as a representative point, and 0 otherwise. $u_{t j}$ takes a value of 1 if $\mathbf{q}^{t}$ is represented by $\mathbf{q}^{j}$ and 0 otherwise. We define the set of representative hypothetical points as $\mathbf{Q}^{*}, \mathbf{Q}^{*}=\left\{\mathbf{q}^{j} \in \mathbf{Q} \mid v_{j}=1\right\}$.

Figure 11 shows $d_{\alpha(\boldsymbol{\lambda})}$-representations of hypothetical points on a MOKP with the following weight sets: $\boldsymbol{\lambda}=(1,0,0), \boldsymbol{\lambda}=(0,1,0)$, and $\boldsymbol{\lambda}=(0,0,1)$. While more hypothetical points are selected in the high-density regions when $\boldsymbol{\lambda}=(1,0,0)$, more hypothetical points close to extreme hypothetical points are selected as representative points when $\boldsymbol{\lambda}=(0,0,1)$, as intended.

Once the representative set of hypothetical points, $\mathbf{Q}^{*} \subseteq \mathbf{Q}$, is found, DBA generates a true nondominated point corresponding to each hypothetical representative point. For each hypothetical representative point $\mathbf{q} \in \mathbf{Q}^{*}$, we search for the true nondominated point, $\mathbf{y}$, that is at minimum Tchebycheff distance using Equation 22 ,

$$
\mathbf{y}=\min _{\mathbf{s} \in \mathbf{\Omega}^{\prime}} \max _{i=1, \ldots, m}\left|s_{i}-q_{i}\right|
$$

We next summarize the steps of DBA: 


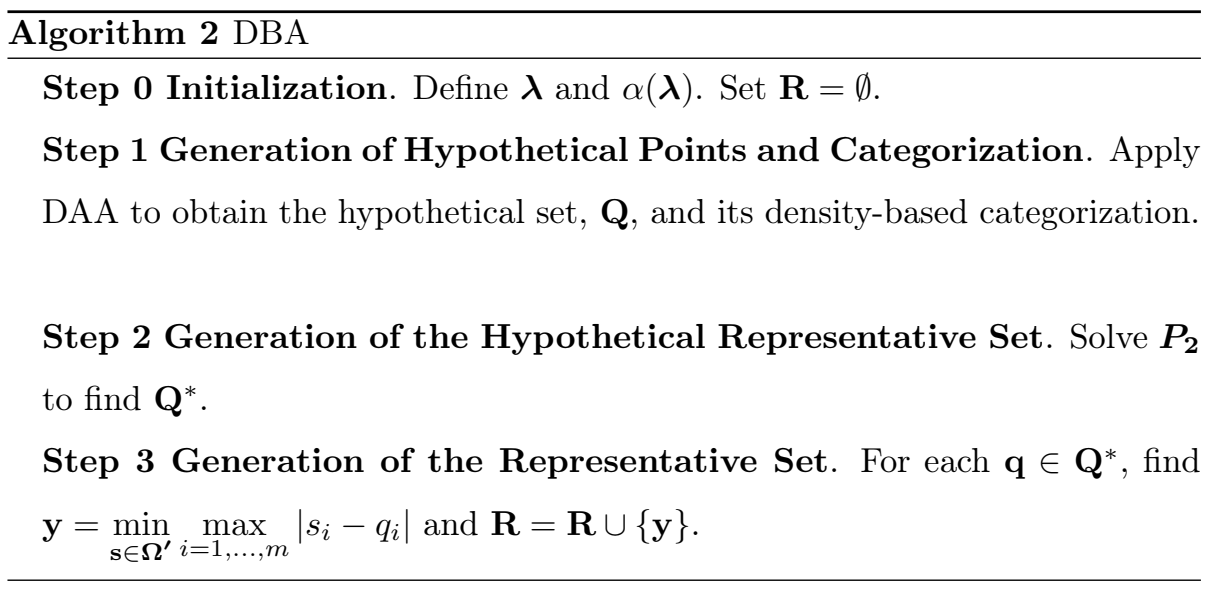

\subsection{Computational Experiments with DBA}

In order to evaluate the performance of DBA, we conduct computational experiments on random instances of MOIPs (see Lokman and Köksalan, 2013. Köksalan and Lokman, 2015). Assume that neither the nondominated set nor the densities of nondominated points are available. Given the desired values of $\boldsymbol{\lambda}$ and $\alpha(\boldsymbol{\lambda})$, we generate the representative set using DAA and we report the resulting weighted coverage gap value. For comparison purposes, we calculate the actual weighted coverage gap, $\alpha_{a}(\boldsymbol{\lambda})$, using the nondominated set and the true categories of the nondominated points. Since the predicted densities may deviate from the true densities as shown in Figure 12, we also calculate the weighted coverage gap value using the predicted densities, $\alpha_{p}(\boldsymbol{\lambda})$. To evaluate how well our algorithm selects the representative hypothetical points, we also consider the case for which the true densities of hypothetical points are available throughout the algorithm, in both representative point selection and weighted coverage gap value calculations. For this case, we report the weighted coverage gap values, $\alpha_{t}(\boldsymbol{\lambda})$. Table 3 presents the weighted coverage gap values for threeobjective MOAPs, MOKPs and MOSPPs having nondominated points in the range $[1014,10701]$ for $\boldsymbol{\lambda}=(1.00,0.75,0.5)$ and $\alpha(\boldsymbol{\lambda})=0.10$.

When $\alpha(\boldsymbol{\lambda})=0.10$, our algorithm produces a representative set with $\alpha_{a}(\boldsymbol{\lambda})=$ 0.1056 on average that is slightly larger than the target level. The resulting 


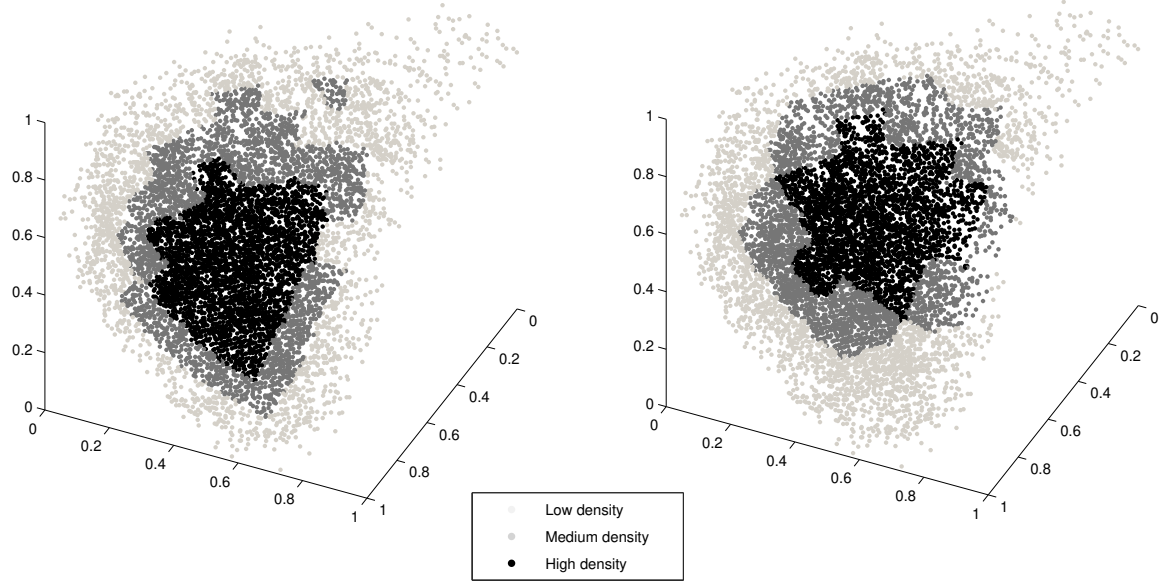

a. True categories based on PWA density values

b. Estimated categories based on DBA

Figure 12: Categorization of the nondominated points for a 3-objective 100-item MOKP

$$
(N=6500, K=3, h=0.2, e=0.1)
$$

weighted coverage gap values in different types MOIPs do not differ much. While $\alpha_{a}(\boldsymbol{\lambda})=0.1056$ on average, the average weighted coverage gap value using the estimated densities is calculated as $\alpha_{p}(\boldsymbol{\lambda})=0.1050$. That is, the estimated categories of the nondominated points are very close to their true categories. We also evaluate the performance of DBA where the true density categories are available throughout the algorithm. In this case, DBA generates a representative set with a weighted coverage value of $\alpha_{t}(\boldsymbol{\lambda})=0.1031$. That is,

Table 3: Summary of Results for $\mathrm{DBA}^{*}(\alpha(\boldsymbol{\lambda})=0.10, \boldsymbol{\lambda}=(1.00,0.75,0.5))$

\begin{tabular}{cccccc}
\hline & $N$ & $|\mathbf{R}|$ & $\alpha_{a}(\boldsymbol{\lambda})$ & $\alpha_{p}(\boldsymbol{\lambda})$ & $\alpha_{t}(\boldsymbol{\lambda})$ \\
\hline $\min$ & 1014.00 & 30.00 & 0.0807 & 0.0775 & 0.0759 \\
$\max$ & 10701.00 & 49.00 & 0.1697 & 0.1697 & 0.1697 \\
$\operatorname{avg}$ & 3973.78 & 36.27 & 0.1056 & 0.1051 & 0.1031 \\
std. dev. & 2115.02 & 3.59 & 0.0188 & 0.0194 & 0.0165 \\
\hline
\end{tabular}

*Values over 51 problems per cell 


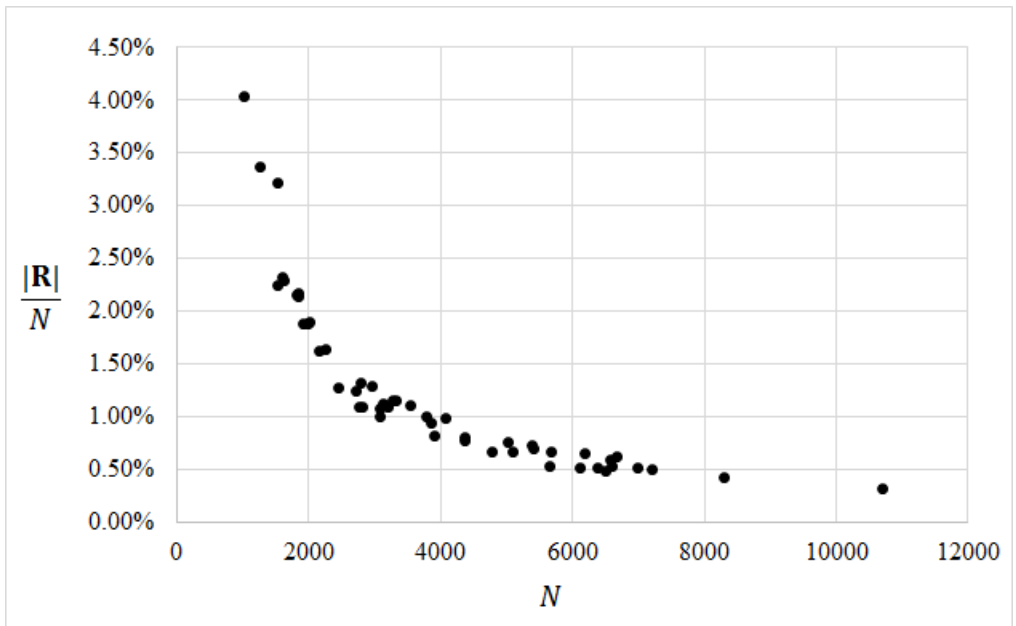

Figure 13: Size of the representative set of DBA with respect to the size of the nondominated set

in the perfect information case where the true densities are known, we would achieve a coverage gap value of 0.1031 . This implies that our density estimation procedure works well since we obtain a coverage gap value close to that of the perfect information case. The reason the perfect information case slightly deviates from the target is due to approximating the efficient set with an $L_{p}$ function.

Figure 13 shows that the computational performance of DBA improves fast as the size of the nondominated set increases. While DBA produces $1.25 \%$ of the points in the nondominated set on average, this ratio decreases up to $0.33 \%$. That is, the ratio of the size of the representative set, $|\mathbf{R}|$, to the size of the nondominated set, $N$, decreases substantially with $N$.

SPA of Ceyhan et al. (2019) is designed to generate representative sets when the desired number of representative points is known a priori. Similar to DBA, it first approximates the nondominated set and then locates the representative points accordingly. However, SPA does not consider the distribution characteristics of the nondominated set in the location of the representative points. Although DBA is designed to capture the distribution properties of the non- 
dominated set and generate representative sets differentiating between different regions, it can also be used in the classical sense without differentiating between different regions as a special case. To see if DBA is competitive with a stateof-the-art algorithm even in this unintended use, we compared its performance with that of SPA on a set of 50-item MOKPs. Since SPA does not differentiate the regions with different density levels, we used equal weights for regions in different categories, $\boldsymbol{\lambda}=(1.00,1.00,1.00)$. The results are summarized in Table 4. Note that SPA aims to minimize the coverage gap value for a given number of representative points while DBA aims to minimize the number of representative points. Since both are approximation methods, it is not possible to control and match the cardinality or actual coverage performance values of the two methods exactly. Therefore, in some instances one algorithm is better than the other in the cardinality and worse in the coverage gap measure. The instances where one algorithm outperforms the other in terms of both cardinality and coverage measures are marked in bold in the Table. These results demonstrate that DBA is competitive even in this unintended application.

\section{Conclusions}

Finding representative sets of nondominated points with desired properties is an important problem for MOIPs since the number of nondominated points grows exponentially with problem size and finding each nondominated point is typically hard. While these desired properties may change from application to application, we observe that the distribution of the nondominated points may be an important property that may be of interest when choosing representative points.

In this study, we search for common characteristics of the distributions of nondominated points in various MOIPs. We introduce a density measure and analyze typical distributions of nondominated points for different MOIPs. Based on our findings, we develop a density-based approximation algorithm, DBA, that approximates the nondominated set using a discrete set of points and categorizes 
Table 4: The performance results of SPA and DBA with $\boldsymbol{\lambda}=(1.00,1.00,1.00)$ on 50-item

\begin{tabular}{|c|c|c|c|c|c|}
\hline \multirow[b]{3}{*}{ Problem } & \multirow[b]{3}{*}{$N$} & \multicolumn{4}{|c|}{ MOKPs } \\
\hline & & \multicolumn{2}{|c|}{ SPA } & \multicolumn{2}{|c|}{ DBA } \\
\hline & & $|\mathbf{R}|$ & $\alpha_{\mathbf{R}}(\boldsymbol{\lambda})$ & $|\mathbf{R}|$ & $\alpha_{\mathbf{R}}(\boldsymbol{\lambda})$ \\
\hline \multirow{3}{*}{1} & \multirow{3}{*}{161} & 5 & 0.1814 & 4 & 0.2222 \\
\hline & & 15 & 0.1757 & 14 & 0.134 \\
\hline & & 25 & 0.0845 & 25 & 0.0956 \\
\hline \multirow{3}{*}{2} & \multirow{3}{*}{496} & 5 & 0.2388 & 5 & 0.1663 \\
\hline & & 10 & 0.109 & 18 & 0.104 \\
\hline & & 50 & 0.101 & 44 & 0.0852 \\
\hline \multirow{3}{*}{3} & \multirow{3}{*}{354} & 5 & 0.1793 & 5 & 0.177 \\
\hline & & 10 & 0.1407 & 12 & 0.1467 \\
\hline & & 25 & 0.1168 & 28 & 0.09 \\
\hline \multirow{3}{*}{4} & \multirow{3}{*}{730} & 5 & 0.2776 & 5 & 0.2057 \\
\hline & & 10 & 0.1481 & 9 & 0.1568 \\
\hline & & 25 & 0.0966 & 36 & 0.0977 \\
\hline \multirow{3}{*}{5} & \multirow{3}{*}{570} & 5 & 0.183 & 7 & 0.1747 \\
\hline & & 10 & 0.1206 & 10 & 0.1372 \\
\hline & & 25 & 0.1414 & 25 & 0.1014 \\
\hline
\end{tabular}

${ }^{*} N$ and $|\mathbf{R}|$ denote the size of the nondominated set and

representative set, respectively.

these points based on their density levels.

In order to generate distribution-based representative sets, we first introduce a new quality measure, weighted coverage gap, that not only considers the representation error of the points but also takes into account their regional characteristics. While DAA is designed to generate a discrete set of hypothetical points that represent the nondominated set and categorize them based on their 
densities, DBA aims to generate a minimum cardinality representative set of the true nondominated points to achieve a desired weighted coverage gap value.

For comparison purposes, we study problems for which the nondominated set is already available but we assume that they are unknown to us in our computational experiments. Our results show that DAA and DBA successfully achieve their goals.

DBA is designed to generate a distribution-based representative set to meet a desired quality level. It aims to minimize the size of the representative set without imposing a limit on it. As a future study, a computational budget can be defined in terms of the number of representative points to minimize the weighted coverage gap under this restriction. Another future study may be to devise and implement different allocation rules for the number of representative points to be selected from each density category.

We believe that the estimation of the densities of nondominated points in different parts of the objective function space is important. This information can be used for various purposes. We demonstrate several uses in conjunction with representative point selection. We believe that our findings will open up a new research direction in the representation of the nondominated sets and future research will further exploit various characteristics of the nondominated set.

\section{Acknowledgement}

This work was supported by the Scientific and Technological Research Council of Turkey (TÜBİTAK), Program: 1001 Grant No: 215M844.

\section{References}

Bektaş T. Disjunctive programming for multiobjective discrete optimisation. INFORMS Journal on Computing 2018;30(4):625-33. 
Boland N, Charkhgard H, Savelsbergh M. A new method for optimizing a linear function over the efficient set of a multiobjective integer program. European journal of operational research 2017;260(3):904-19.

Ceyhan G, Köksalan M, Lokman B. Finding a representative nondominated set for multi-objective mixed integer programs. European Journal of Operational Research 2019;272(1):61-77.

Costa M, Minisci E. Moped: a multi-objective parzen-based estimation of distribution algorithm for continuous problems. In: International Conference on Evolutionary Multi-Criterion Optimization. Springer; 2003. p. 282-94.

Dächert K, Klamroth K. A linear bound on the number of scalarizations needed to solve discrete tricriteria optimization problems. Journal of Global Optimization 2015;61(4):643-76.

Dächert K, Klamroth K, Lacour R, Vanderpooten D. Efficient computation of the search region in multi-objective optimization. European Journal of Operational Research 2017;260(3):841-55.

Dhaenens C, Lemesre J, Talbi EG. K-ppm: A new exact method to solve multi-objective combinatorial optimization problems. European Journal of Operational Research 2010;200(1):45-53.

Duda RO, Hart PE, Stork DG. Pattern classification. Wiley, New York, 1973.

Ehrgott M, Gandibleux X. A survey and annotated bibliography of multiobjective combinatorial optimization. OR Spektrum 2000;22:425-60.

Ehrgott M, Gandibleux X. Approximative solution methods for multiobjective combinatorial optimization. Top 2004;12(1):1-63.

Ehrgott M, Gandibleux X, Przybylski A. Exact methods for multi-objective combinatorial optimisation. In: Multiple criteria decision analysis. Springer; 2016. p. $817-50$. 
Faulkenberg SL, Wiecek MM. On the quality of discrete representations in multiple objective programming. Optimization and Engineering 2010;11(3):42340.

Figueira JR, Fonseca CM, Halffmann P, Klamroth K, Paquete L, Ruzika S, Schulze B, Stiglmayr M, Willems D. Easy to say they are hard, but hard to see they are easy - towards a categorization of tractable multiobjective combinatorial optimization problems. Journal of Multi-Criteria Decision Analysis 2017;24(1-2):82-98.

Karasakal E, Köksalan M. Generating a Representative Subset of the Nondominated Frontier in Multiple Criteria Decision Making. Operations Research 2009;57(1):187-99.

Kirlik G, Saym S. A new algorithm for generating all nondominated solutions of multiobjective discrete optimization problems. European Journal of Operational Research 2014;232(3):479-88.

Köksalan M, Lokman B. Approximating the nondominated frontiers of multiobjective combinatorial optimization problems. Naval Research Logistics (NRL) 2009;56(2):191-8.

Köksalan M, Lokman B. Finding nadir points in multi-objective integer programs. Journal of Global Optimization 2015;62(1):55-77.

Köksalan MM. A heuristic approach to bicriteria scheduling. Naval Research Logistics (NRL) 1999;46(7):777-89.

Laumanns M, Thiele L, Zitzler E. An efficient, adaptive parameter variation scheme for metaheuristics based on the epsilon-constraint method. European Journal of Operational Research 2006;169(3):932-42.

Lokman B, Köksalan M. Finding all nondominated points of multi-objective integer programs. Journal of Global Optimization 2013;57(2):347-65. 
Lokman B, Köksalan M. Finding highly preferred points for multi-objective integer programs. IIE Transactions 2014;46(11):1181-95.

Masin M, Bukchin Y. Diversity Maximization Approach for Multiobjective Optimization. Operations Research 2008;56(2):411-24.

Mavrotas G, Florios K. An improved version of the augmented $\varepsilon$-constraint method (augmecon2) for finding the exact pareto set in multi-objective integer programming problems. Applied Mathematics and Computation 2013;219(18):9652-69.

Özlen M, Azizoğlu M. Multi-objective integer programming: A general approach for generating all non-dominated solutions. European Journal of Operational Research 2009;199(1):25-35.

Özlen M, Burton BA, MacRae CA. Multi-objective integer programming: An improved recursive algorithm. Journal of Optimization Theory and Applications 2014;160(2):470-82.

Parzen E. On estimation of a probability density function and mode. The annals of mathematical statistics 1962;33(3):1065-76.

Przybylski A, Gandibleux X, Ehrgott M. A two phase method for multiobjective integer programming and its application to the assignment problem with three objectives. Discrete Optimization 2010;7(3):149-65.

Sayın S. Measuring the quality of discrete representations of efficient sets in multiple objective mathematical programming. Mathematical Programming 2000;87(3):543.

Sylva J, Crema A. A method for finding the set of non-dominated vectors for multiple objective integer linear programs. European Journal of Operational Research 2004;158(1):46-55.

Sylva J, Crema A. A method for finding well-dispersed subsets of non-dominated vectors for multiple objective mixed integer linear programs. European Journal of Operational Research 2007;180(3):1011-27. 
Vincent P, Bengio Y. Manifold parzen windows. In: Advances in neural information processing systems. 2003. p. 849-56. 